\title{
Carnosic acid protects against lipopolysaccharide-induced acute lung injury in mice
}

\author{
QUAN LI ${ }^{1}$, LING LIU ${ }^{2}$, HAIJUN SUN ${ }^{1}$ and KUNYUE CAO ${ }^{1}$ \\ ${ }^{1}$ Intensive Care Unit, Suqian First Hospital, Suqian, Jiangsu 223800; ${ }^{2}$ Intensive Care Unit, \\ Zhongda Hospital Southeast University, Nanjing, Jiangsu 210009, P.R. China
}

Received May 23, 2018; Accepted May 31, 2019

DOI: $10.3892 /$ etm.2019.8042

\begin{abstract}
Acute respiratory distress syndrome is a well-known inflammatory disease associated with high rates of morbidity and mortality due to a lack of effective treatment methods. Carnosic acid (CA) is a phenolic diterpene compound that serves a central role in cytoprotective responses to inflammation. In the present study, the protective mechanism of CA on acute lung injury (ALI) induced by lipopolysaccharide (LPS) was investigated. Mice were randomly assigned to the following five groups: Control group, LPS group, and LPS plus CA groups (at 10, 20 and $40 \mathrm{mg} / \mathrm{kg}$ doses). Following pre-treatment with vehicle or CA,ALI was induced by the administration of LPS. At $6 \mathrm{~h}$ after LPS treatment, mice were sacrificed and lung tissues were harvested for histologic analysis and the determination of wet-to-dry ratio, myeloperoxidase activity and toll-like receptor 4 (TLR4) and NF- $\kappa \mathrm{B}$ expression. Additionally, the levels of interleukin (IL)-1 $\beta$, IL-6 and tumor necrosis factor- $\alpha$ (TNF- $\alpha$ ) were determined in bronchoalveolar lavage fluid (BALF) and lung tissues, as well as the rate of apoptosis of the isolated neutrophils from BALF. The alleviation of LPS-induced ALI by $\mathrm{CA}$ was confirmed by histologic results and a reduction in the wet-to-dry ratio of lung tissues. Additionally, CA was revealed to significantly suppress the inhibitory effect of LPS on neutrophil apoptosis and the promoting effects of LPS on IL-1 $\beta$, IL-6, TNF- $\alpha$, TLR4 and NF- $\kappa$ B expression, and NF- $\kappa$ B phosphorylation. The current results indicated that $\mathrm{CA}$ protects against LPS-induced ALI via a mechanism that inhibits inflammation.
\end{abstract}

\section{Introduction}

Acute respiratory distress syndrome (ARDS) is a severe inflammatory disease of the lungs, characterized by hypoxemia that is caused by the severe impairment of gas exchange and lung

Correspondence to: Dr Quan Li, Intensive Care Unit, Suqian First Hospital, 120 Suzhi Road, Sucheng, Suqian, Jiangsu 223800, P.R. China

E-mail: liquanzz2012@126.com

Key words: acute respiratory distress syndrome, carnosic acid, inflammation, NF- $\mathrm{KB}$ mechanics as a result of acute lung injury (ALI) and edema (1). With an increasing incidence of 200,000 cases annually in the United States, ARDS has a high rate of mortality (30-50\%) and is responsible for exorbitant health care expenditures (1-3). While several factors are known to induce ARDS, including hyperoxia and hemorrhage, sepsis and pneumonia are the leading causes of the disease (2). Although a variety of interventions and treatments have been developed for this inflammatory disorder, the mortality rate of ARDS remains higher than acceptable (4). Therefore, there is an urgent need to develop an effective method or drug for treating ARDS.

Lipopolysaccharides (LPSs) located in the outer membrane of gram-negative bacteria have been reported to cause ARDS by increasing inflammatory cytokine production in lung tissue (5). As an important component of the inflammatory system, neutrophils partially initiates inflammation in patients with ALI/ARDS and contributes to the development of ARDS-associated pulmonary edema (6). After infiltrating the lungs and migrating into the airways, neutrophils secrete various pro-inflammatory cytokines, including tumor necrosis factor- $\alpha$ (TNF- $\alpha$ ), interleukin (IL)- $1 \beta$ and IL-6, which disrupt endothelial and epithelial barriers (6-8). It has been reported that the increased expression of proinflammatory cytokines can be ascribed to the $\mathrm{NF}-\kappa \mathrm{B}$ pathway (2). Activation of the NF- $\kappa \mathrm{B}$ pathway is dependent on the phosphorylation of NF- $\kappa \mathrm{B}$ and its subsequent translocation to the nucleus, resulting in gene transcription $(2,9)$. Proinflammatory signaling and resultant immune responses are triggered by toll-like receptors (TLRs), which are highly conserved pattern recognition receptors that recognize nonendogenous pathogen-associated molecular pattern molecules (1). Ten functional TLRs have been identified in humans, and appear to be associated with ARDS (10). TLR3 was revealed to participate in hyperoxia-induced ARDS and TLR2 was demonstrated to mediate hemorrhage-induced ARDS (11,12). TLR4 was also identified as a pivotal regulator capable of inducing ARDS in various murine models, including those induced by sepsis $(12,13)$.

Recent reports have demonstrated that certain plant extracts help to protect against inflammation-associated diseases $(14,15)$. For example, phytochemicals present in ginkgo biloba, sun ginseng and blackberries have been shown to have therapeutic potential for endotoxin-associated diseases (16). Carnosic acid (CA) is a benzenediol abietane diterpene found in a variety of herbal plants including rosemary and sage (17). It is an antioxidant that has multiple pharmacological effects, 
including anti-obesity, anti-inflammatory, anti-tumor and neuroprotective activities (18-21). CA has been revealed to protect against myocardial injury and inhibit colitis in vivo, potentially via anti-inflammatory activity (22). However, to the best of our knowledge, no studies have investigated the effects of CA on ARDS.

In the present study, the effect of CA on LPS-induced ALI in mice was investigated. The impacts on lung injury and inflammation, as well as neutrophil infiltration and the generation of TNF- $\alpha$, IL- $1 \beta$ and IL- 6 were determined. The ability of CA to inhibit the NF- $\kappa$ B pathway, which is critically associated with neutrophil activation and inflammatory responses, was also assessed.

\section{Materials and methods}

Animals and treatments. Male BALB/c mice (age, $~ 8$ weeks; weight, 21-25 g) were purchased from the Model Animal Research Center of Nanjing University and housed in a standard laboratory room maintained at $20-26^{\circ} \mathrm{C}$ and $40-70 \%$ relative humidity, under a $12 \mathrm{~h}$ light/dark cycle. Mice also received free access to water and food.

Mice were randomly assigned to 5 groups ( $n=6 /$ group). A group of mice was used as control, while mice the other 4 groups were used to establish an acute lung injury (ALI) model induced with LPS (Sigma-Aldrich; Merck KGaA). At $\sim 12 \mathrm{~h}$ prior to receiving an intravenous (IV) injection of LPS ( $4 \mathrm{mg} / \mathrm{kg}$ ), the mice were given CA at the following doses: 0 , 10,20 or $40 \mathrm{mg} / \mathrm{kg}$. A total of $6 \mathrm{~h}$ later, the mice were sacrificed and samples of lung tissue were removed for testing. CA was purchased from Dalian Meilun Biotech Co., Ltd, dissolved in $0.5 \% \mathrm{v} / \mathrm{v}$ DMSO in saline and injected into mice intraperitoneally. Bronchoalveolar lavage fluid (BALF) was obtained for MPO analysis, and the left lungs were collected for histopathological examination, while the, right lungs were collected for wet-to-dry (W/D) analysis, western blotting and reverse transcription quantitative PCR (RT-qPCR) examinations. All animal experiments were performed at the Zhongda Hospital Southeast University (Nanjing, China), and all experimental protocols were approved by the Ethics Committee of the Zhongda Hospital Southeast University.

Bronchoalveolar lavage fluid and tissue sampling. Each mouse was anesthetized with $0.75 \%$ pentobarbital $(35 \mathrm{mg} / \mathrm{kg}$; IV) followed by decapitation. Animals were confirmed dead when no breathing or heart beat was detected. Following sacrifice, neck skin was dissected and the trachea was rapidly exposed. Next, a catheter was deeply inserted into the trachea and the lungs were lavaged 3 times with $1 \mathrm{ml}$ of cold PBS solution. The BALF collected from each mouse was centrifuged at $420 \mathrm{x} \mathrm{g}$ for $15 \mathrm{~min}$ at $4^{\circ} \mathrm{C}$ and the supernatant was stored at $-80^{\circ} \mathrm{C}$ until further use. Cell pellets were re-suspended in PBS for subsequent neutrophil purification. The lungs were cut into two parts, placed into individual centrifuge tubes, snap frozen in liquid nitrogen and stored at $-80^{\circ} \mathrm{C}$. Lung tissue $(\sim 100 \mathrm{mg})$ were placed into centrifuge tubes and suspended in cool normal saline solution. Tissue samples were then homogenized with homogenate rods and centrifuged at $12,000 \times \mathrm{g}$ for $10 \mathrm{~min}$ at $4^{\circ} \mathrm{C}$ to collect supernatant for myeloperoxidase (MPO), ELISA analysis and western blotting.
Neutrophil purification and apoptosis analysis. Neutrophils were isolated by discontinuous Percoll gradient centrifugation as previously described (23). In brief, $2 \mathrm{ml}$ of Percoll solution diluted with HBSS (1,090 mg/ml; Sigma-Aldrich; Merck KGaA) was added to a tube and another $2 \mathrm{ml}$ of Percoll solution $(1,080 \mathrm{mg} / \mathrm{ml})$ was gently layered on top of the first solution. Resuspended cells were then gently layered onto the Percoll solution, and centrifuged at $960 \mathrm{x} \mathrm{g}$ for $15 \mathrm{~min}$ at $4^{\circ} \mathrm{C}$. The neutrophil layer located between the Percoll layers was collected into tubes containing PBS, which were then centrifuged at $420 \mathrm{x}$ g for $5 \mathrm{~min}$ at $4^{\circ} \mathrm{C}$. The cells were subsequently resuspended in pre-cooled PBS and cells were fixed overnight at $4{ }^{\circ} \mathrm{C}$ in pre-cooled $70 \%$ ethanol. After washing twice with PBS, fixed cells were incubated with $5 \mu \mathrm{l}$ Annexin V-FITC and $10 \mu \mathrm{l}$ propidium iodide (PI; cat. no. AP101-30; Hangzhou Lianke Biology Technology Co., Ltd.) at $4^{\circ} \mathrm{C}$ for $30 \mathrm{~min}$ in the dark and then analyzed for apoptosis using a BD FACSCalibur flow cytometer (BD Biosciences). Data were analyzed using FlowJo software (Version 7.6; FlowJo, LLC).

ELISA. ELISA kits (Biolegend, Inc.) were used to measure the levels of IL-1 $\beta$ (cat. no. 432601), IL-6 (cat. no. 431304) and TNF- $\alpha$ (cat. no. 430904) in BALF and lung tissue homogenates according to the manufacturer's protocols. Samples and standards were pipetted into a microplate pre-coated with capture antibodies (provided as part of the aforementioned ELISA kits) and incubated at room temperature for $2 \mathrm{~h}$. After washing 3 times with $300 \mu \mathrm{l}$ Wash Buffer, biotinylated detection antibodies (all provided as part of the aforementioned kits) were added and incubated for 1 hat room temperature, followed by incubation with avidin horseradish peroxidase (HRP) conjugate for $30 \mathrm{~min}$. The immune reaction was visualized by the addition of 3,3',5,5'-Tetramethylbenzidine (Beyotime Institute of Biotechnology) substrate solution followed by incubation at room temperature in the dark for $15 \mathrm{~min}$. Subsequently, the reaction was stopped by the addition of $1 \mathrm{M} \mathrm{H}_{2} \mathrm{SO}_{4}$ and the optical density of each well was measured at $450 \mathrm{~nm}$ with a microplate reader (TECAN-GENious; Tecan Group, Ltd.).

Lung myeloperoxidase activity determination. MPO activity in lung homogenates was determined using a MPO assay kit according to the manufacturer's protocol (cat. no. A044-1-1; Nanjing Jiancheng Bioengineering Institute). The homogenates were subsequently centrifuged at 5,000 x g for $10 \mathrm{~min}$ at $4^{\circ} \mathrm{C}$ and the supernatants were collected and used to calculate MPO activity based on the maximal absorbance at $532 \mathrm{~nm}$. The homogenates were mixed with reagent II, followed by the addition of reagent IV and a chromogenic agent, and left to react for $10 \mathrm{~min}$ at $60^{\circ} \mathrm{C}$. MPO activity was calculated based on the maximal absorbance at $532 \mathrm{~nm}$ and activity was normalized to the total protein concentration of the same sample, which was determined using a bicinchoninic acid assay (cat. no. 23225; Pierce; Thermo Fisher Scientific, Inc.).

Lung wet-to-dry weight (W/D) ratio. To evaluate lung edema, mice were euthanized and the right lungs were collected, weighed and placed in an oven at $70^{\circ} \mathrm{C}$ for $48 \mathrm{~h}$. Dried tissues were then weighed to calculate the W/D ratio. 
Histopathological and immunohistochemical examinations. The left lungs of mice were fixed in $10 \%$ buffered formalin for $24 \mathrm{~h}$ at room temperature. A small section of fixed lung tissue was cut with a surgical blade, dehydrated in a series of graded ethanol solutions $(30,60,80,95$ and $100 \%)$, cleared with xylene and embedded inparaffin. Embedded tissue was subsequently cut into $5 \mu \mathrm{m}$ sections, which were deparaffinized with xyleneand rehydrated in a descending gradient of ethanol concentrations $(100,95,80,60$ and 30\%). Some sections were stained with hematoxylin and eosinat room temperature for $15 \mathrm{~min}$ and observed under a light microscope (magnification, x400), while other sections were used for immunohistochemical examinations of TLR4 and NF- $\mathrm{NB}$ expression. Endogenous tissue peroxidase was inactivated by incubation with $0.5 \% \mathrm{H}_{2} \mathrm{O}_{2}$ for $5 \mathrm{~min}$ at room temperature, and antigen retrieval was performed by boilingtissue in citrate buffer ( $\mathrm{pH} \mathrm{6.0)} \mathrm{for} 10 \mathrm{~min}$. Tissue sections were then blocked with $1 \%$ BSA (Beijing Solarbio Science \& Technology Co., Ltd.) for $30 \mathrm{~min}$ at room temperature and then incubated for $1 \mathrm{~h}$ at room temperature with primary antibodies against TLR4 (cat. no. sc-293072; 1:200; Santa CruzBiotechnology, Inc.) and NF-кB (cat. no. sc-514451; 1:200; Santa Cruz Biotechnology, Inc.). Sections were then incubated for $20 \mathrm{~min}$ at room temperature with HRP-conjugated secondary antibodies (cat. no. IH-0031; 1:5,000; Beijing Dingguo Changsheng Biotechnology Co., Ltd.). Color was developed with a diaminobenzidine kit (cat. no. JD091-1G; Beijing Dingguo Changsheng Biotechnology Co., Ltd.), followed by counterstaining with hematoxylin for $2 \mathrm{~min}$ at room temperature.

Reverse transcription quantitative PCR. Total RNA was extracted from the collected mouse lung tissues using TRIzol reagent (Takara Biotechnology Co., Ltd.) and $3 \mu \mathrm{g}$ of total RNA was reverse transcribed into cDNA using a Bestar $^{\mathrm{TM}}$ qPCR RT kit (cat. no. DBI-2220; DBI Bioscience) according to the manufacturer's protocol. qPCR was performed using Bestar ${ }^{\mathrm{TM}}$ SYBRGreen qPCR MasterMix (cat. no. DBI-2044; DBI Bioscience) in an ABI 7500 RT-PCR system (Applied Biosystems; Thermo Fisher Scientific, Inc.). The thermo cycling conditions were as follows: Denaturation at $95^{\circ} \mathrm{C}$ for $10 \mathrm{~min}$, followed by 40 cycles of $94^{\circ} \mathrm{C}$ for $20 \mathrm{sec}$, $58^{\circ} \mathrm{C}$ for $20 \mathrm{sec}$ and $72^{\circ} \mathrm{C}$ for $20 \mathrm{sec}$. The primers used were as follows: TLR4 forward, 5'-CCATTGCTTGGCGAATGTTT-3' and reverse, 5'-TGTCTCAGGCTGTTTGTTCC-3'; NF-кB forward, 5'-AGAGCAACCAAAACAGAGGG-3' and reverse, 5'-TGCAAATTTTGACCTGTGGG-3'; $\beta$-actin forward, 5'-CATTGCTGACAGGATGCAGA-3' and reverse, 5'-CTG CTGGAAGGTGGACAGTGA-3'. The levels of target mRNA were analyzed using the $2^{-\triangle \Delta C q}$ method (24) and normalized using $\beta$-actin as a control. Each test was repeated three times.

Western blot analysis. Collected lung tissues were lysed with RIPA buffer (Beyotime Institute of Biotechnology) supplemented with a protease inhibitor cocktail (Sigma-Aldrich; Merck KGaA). The protein concentrations in the lysates were determined using a BCA kit (cat. 23225; Pierce; Thermo Fisher Scientific, Inc.). Equal quantities of protein $(20 \mu \mathrm{g})$ from the lysates were separated using SDS-PAGE (10\% gel) and the separated protein bands were transferred onto polyvinylidene difluoride membranes (Bio-Rad Laboratories, Inc.), which were subsequently blocked with 5\% non-fat dry milk in TBST
(Tris-buffered saline with $0.1 \%$ Tween-20) at room temperature for $1 \mathrm{~h}$. The membranes were then washed and incubated overnight at $4^{\circ} \mathrm{C}$ with primary antibodies against TLR4 (cat. no. sc-52962; 1:1,000; Santa Cruz Biotechnology, Inc.), NF-кB (cat. no. sc-514451; 1:200; Santa Cruz Biotechnology, Inc.), p-NF- $\kappa \mathrm{B}$ (cat. no. sc-135768; 1:1,000; Santa Cruz Biotechnology, Inc.) and GAPDH (cat. no. ab8245; 1:2,000; Abcam). The antibody against $\mathrm{NF}-\kappa \mathrm{B}$ or $\mathrm{p}-\mathrm{NF}-\kappa \mathrm{B}$ recognized the p65 or p-p65 subunit, respectively. Membranes were subsequently washed with TBST and then incubated with HRP-conjugated secondary antibodies at $37^{\circ} \mathrm{C}$ for $1 \mathrm{~h}$ (cat. no. BA1050; 1:5,000; Boster Biological Technology). Immunostained protein bands were visualized with a chemiluminescent substrate (ECL-Plus; GE Healthcare). The assay was repeated three times. Semi-quantitative analysis was performed using Image-Pro Plus 6.0 software (Media Cybernetics, Inc.).

Statistical analysis. Data are expressed as the mean \pm standard deviation. One-way ANOVA and a two-tailed Student's t-test were used to compare the differences in parameters between experimental groups. All statistical analyses were performed using IBM SPSS Statistics for Windows, version 21.0 (IBM Corp.) and $\mathrm{P}<0.05$ was considered to indicate a statistically significant difference.

\section{Results}

Effect of CA on LPS-induced ALI. To observe the effect of CA on lung injuries caused by LPS, the lungs were collected $6 \mathrm{~h}$ after LPS treatment and subjected to a histologic examination. As presented in Fig. 1A, lungs in the control group exhibited no signs of hemorrhage or edema and little evidence of inflammatory cell infiltration. In contrast, LPS treatment alone induced hemorrhage and pathologic alterations typically associated with ALI, including hemorrhage, accumulation of inflammatory cells in the alveolar space, alveolar wall thickening and edema of the lung interstitium and alveoli, as indicated with black arrows (Fig. 1A). Consistent with the afore mentioned histopathological observations, the W/D ratio was significantly increased after LPS treatment (Fig. 1B). However, the increase induced by LPS was significantly inhibited by CA treatment at 20 and $40 \mathrm{mg} / \mathrm{kg}$, indicating that CA protected the lungs from injuries induced by LPS.

Effect of CA on neutrophil apoptosis in LPS-induced ALI. Neutrophils are an important contributor to ARDS (25). To compare the viability of neutrophils, cell apoptosis was determined via flow cytometry. As presented in Fig. 2, LPS treatment significantly decreased cell apoptosis compared with the control. In mice that received CA at doses of 20 and $40 \mathrm{mg} / \mathrm{kg}$, apoptosis was significantly increased compared with that of the LPS group. Additionally, the rate of apoptosis in the CA-treated groups increased with higher doses and gradually approached the rate of apoptosis observed in the control group. At $40 \mathrm{mg} / \mathrm{kg}$, there was no statistical difference from the control group. These results indicated that CA treatment significantly inhibited the suppression of neutrophil apoptosis by LPS.

Effect of CA on MPO activity and IL-1 $\beta, I L-6$ and TNF- $\alpha$ levels. In the present study, MPO activity was used as an 


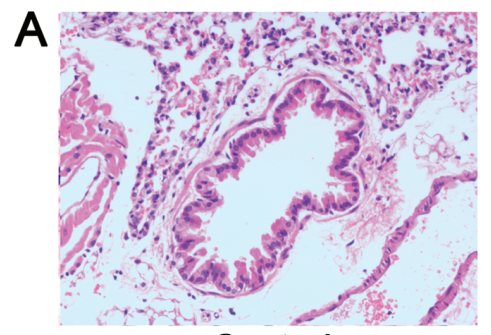

Control

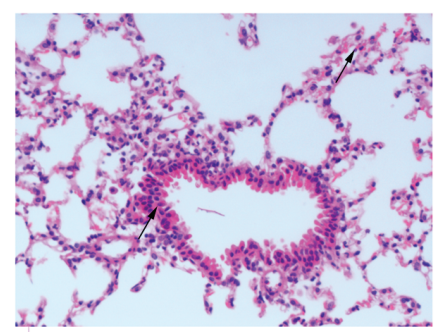

LPS+20 $\mathrm{mg} / \mathrm{kg} \mathrm{CA}$

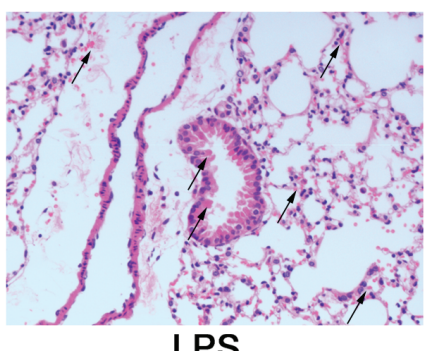

LPS

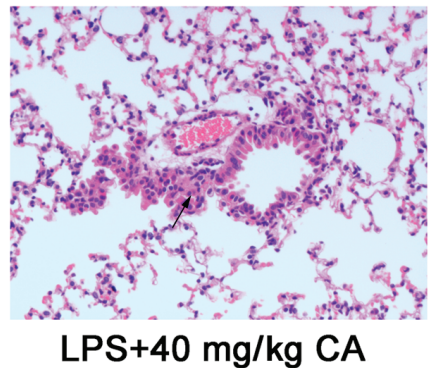

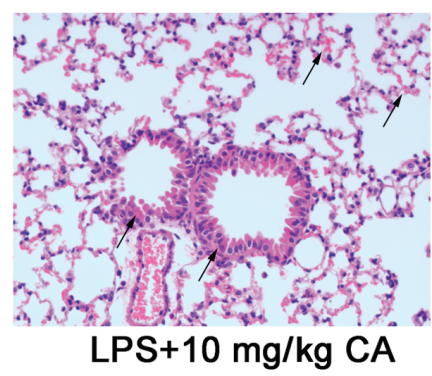

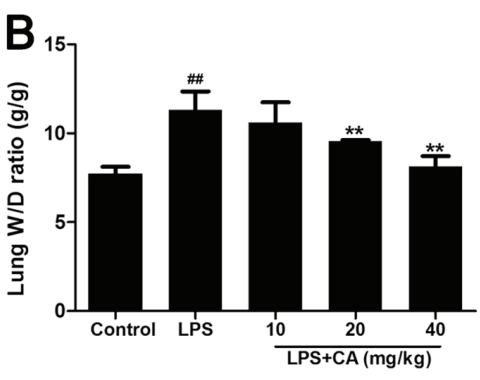

Figure 1. Injury of lung tissue following exposure to LPS and CA pre treatment. Mice were treated with CA ( $0,10,20$ and 40 mg/kg), followed by exposure to LPS (4 mg/kg) or the vehicle control (PBS) $12 \mathrm{~h}$ later. At $6 \mathrm{~h}$ post LPS/control treatment, the mice were sacrificed for histopathological analysis and edema evaluation. (A) Hematoxylin and eosin staining of lung tissue. No abnormal findings were noted in the control group. Hemorrhage and inflammatory cell infiltration were observed in the LPS-treated groups, but were attenuated by CA (magnification, x400; indicated with black arrows). (B) W/D ratio of lungs following LPS and CA treatment. ${ }^{\# \#} \mathrm{P}<0.01$ vs. the control group; ${ }^{* *} \mathrm{P}<0.01$ vs. the LPS-treated group. LPS, lipopolysaccharide; CA, carnosicacid; W/D, wet to dry.
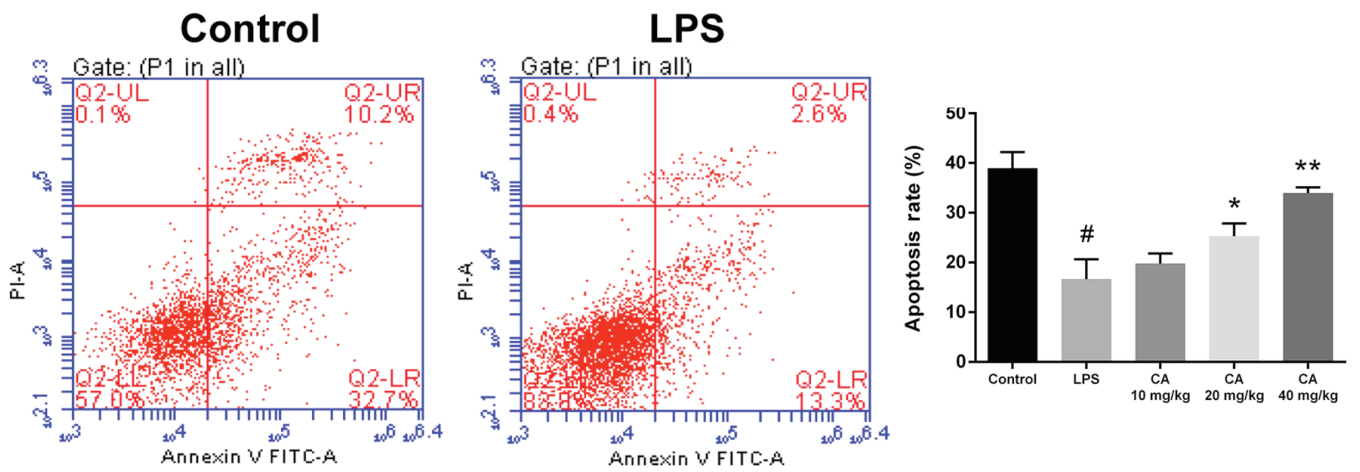

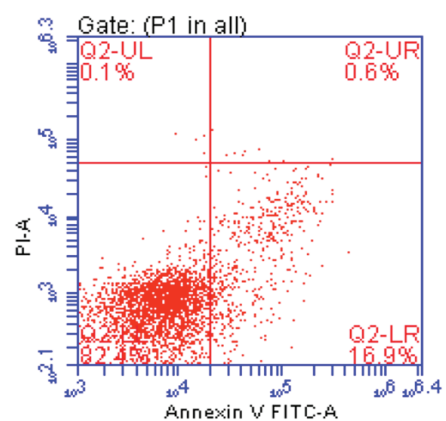

10

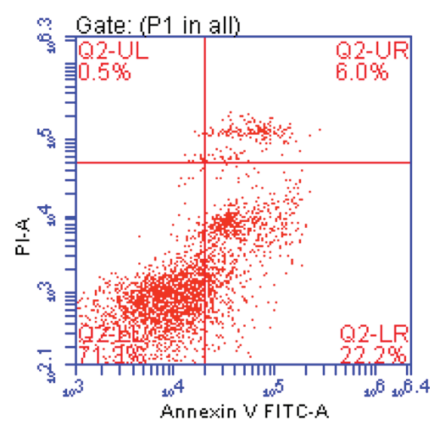

20

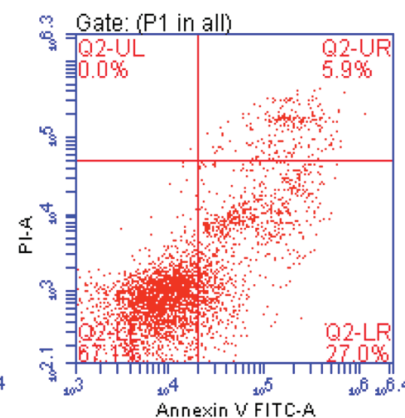

40

\section{LPS+CA (mg/kg)}

Figure 2. Apoptosis of neutrophils isolated via Percoll gradient from bronchoalveolar lavage fluid following exposure to LPS (4 mg/kg) and CA (0, 10 , 20 or $40 \mathrm{mg} / \mathrm{kg}$ ) pretreatment. ${ }^{\#} \mathrm{P}<0.01$ vs. the control group; ${ }^{*} \mathrm{P}<0.05$ and ${ }^{* *} \mathrm{P}<0.01$ vs. the LPS-treated group. LPS, lipopolysaccharide; CA, carnosicacid; PI, propidium iodide.

indicator of neutrophil infiltration. As presented in Fig. 3A, significantly increased MPO activity was observed in the LPS group, when compared with MPO activity in the control group. Pretreatment with CA significantly reduced MPO activity in LPS-challenged lungs in a dose-dependent manner.
These results indicated that LPS induced the infiltration of neutrophils into lung tissue and that the effect was significantly inhibited by CA treatment.

After confirming neutrophil infiltration, the levels of IL-1 $\beta$, IL- 6 and TNF- $\alpha$ were determined to investigate the effect of 
A

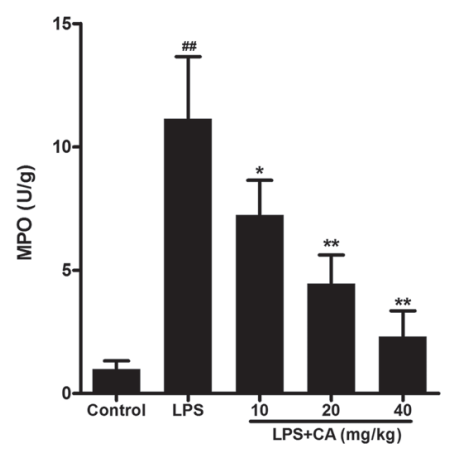

B
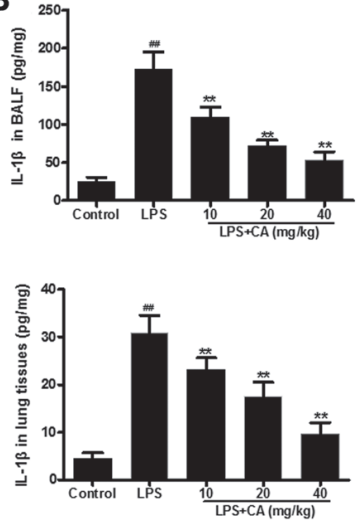
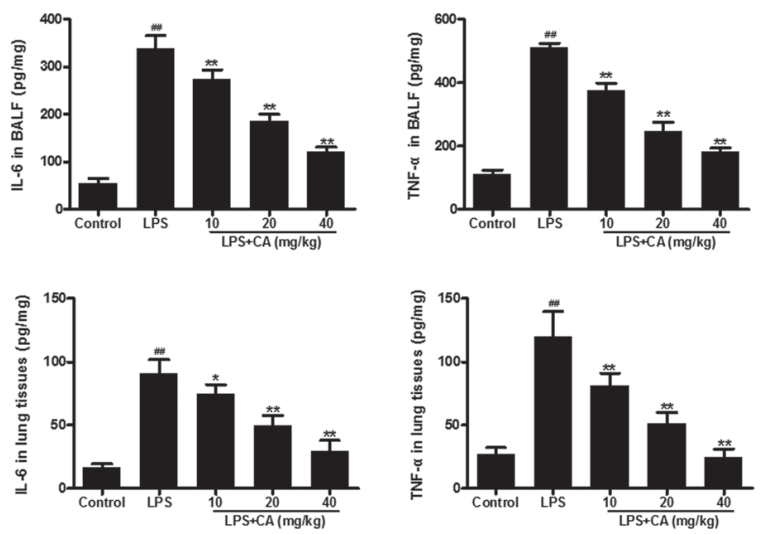

Figure 3. Inflammatory markers in BALF and lung tissue following exposure to LPS (4 mg/kg) and CA ( $(0,10,20 \mathrm{or} 40 \mathrm{mg} / \mathrm{kg})$ pretreatment. (A) MPO activity in BALF. (B) Levels of IL-1 $\beta$, IL-6 and TNF- $\alpha$ in BALF and lung tissue as determined by ELISA. ${ }^{\# \#} \mathrm{P}<0.01$ vs. the control group; ${ }^{*} \mathrm{P}<0.05$ and ${ }^{* * *} \mathrm{P}<0.01$ vs. the LPS-treated group. BALF, bronchoalveolar lavage fluid; LPS, lipopolysaccharide; CA, carnosicacid; MPO, myeloperoxidase; IL, interleukin; TNF- $\alpha$, tumor necrosis factor $\alpha$.

A

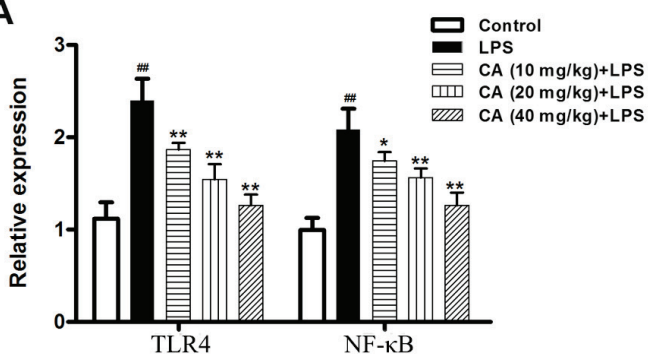

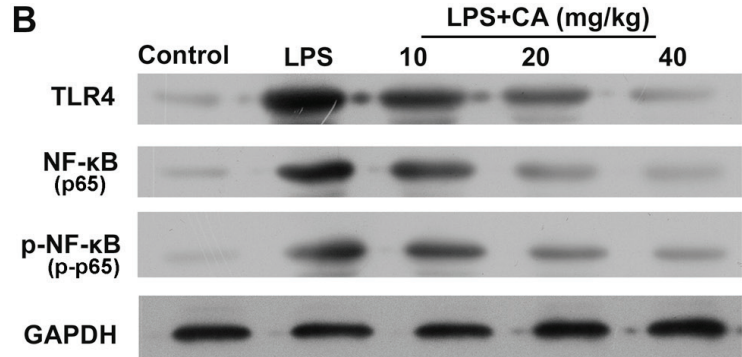

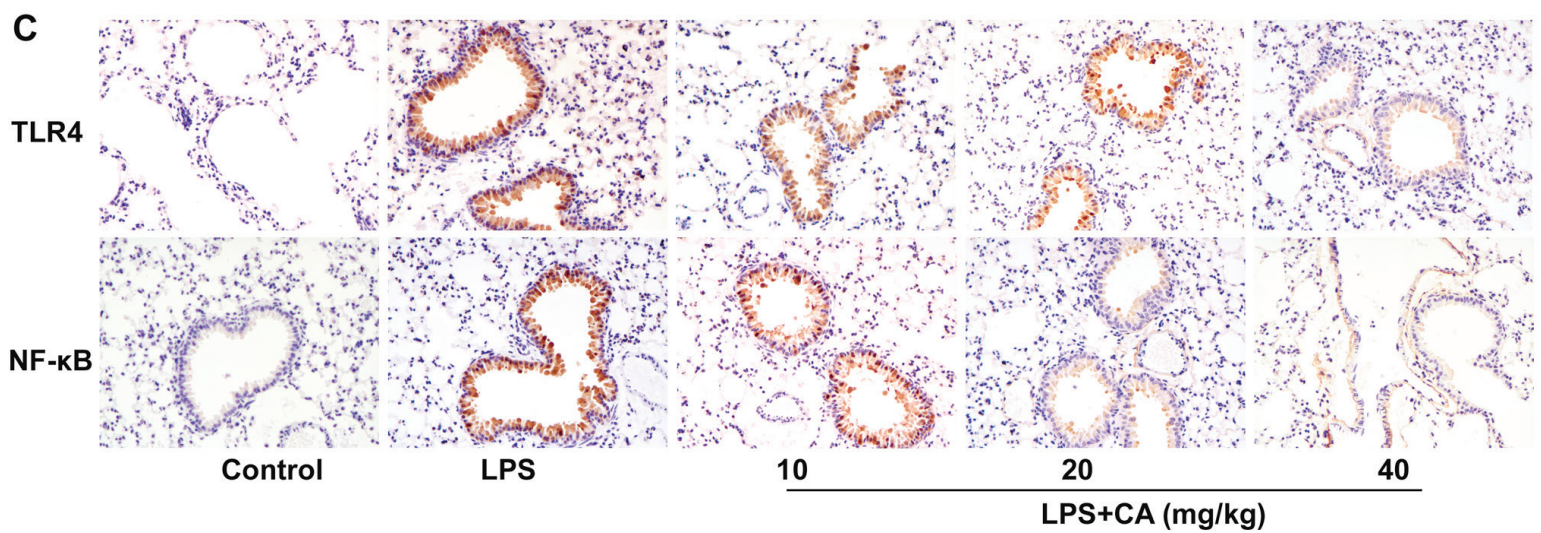

Figure 4. TLR4 and NF- $\mathrm{B}$ expression in lung tissue following exposure to LPS (4 mg/kg) and CA (0, 10, 20 or $40 \mathrm{mg} / \mathrm{kg})$ pretreatment. (A) TLR4 and NF- $\mathrm{B}$ mRNA expression in lung tissue as determined by reverse transcription-quantitative PCR. ${ }^{\# \#} \mathrm{P}<0.01$ vs. the control group; ${ }^{*} \mathrm{P}<0.05$ and ${ }^{* *} \mathrm{P}<0.01$ vs. the LPS-treated group. (B) TLR4, total p65 and p-p65 levels as determined by western blotting. (C) TLR4 and P65 expression in lung tissue as determined by immunohistochemistry (magnification, x200). LPS, lipopolysaccharide; CA, carnosicacid; TLR4, toll-like receptor 4; p65, p65 subunit of NF- $\mathrm{B}$; p-p65, phosphorylated p65.

CA on LPS-induced inflammation. As presentedin Fig. 3B, the levels of IL-1 $\beta$, IL- 6 and TNF- $\alpha$ in the BALF and lung tissues of the LPS group were significantly increased compared with those in the control group. Pretreatment with CA significantly reduced IL- $1 \beta$, IL- 6 and TNF- $\alpha$ levels in LPS-challenged lungs in a dose-dependent manner. These results indicated that LPS induced inflammation in the lungs and that this inflammation could be significantly inhibited by CA treatment.

Effects of CA on TLR4 and NF- $\kappa B$ expression. TLR4 and $\mathrm{NF}-\kappa \mathrm{B}$ serve critical roles in LPS-induced inflammation (26). In the present study, the expression of these inflammatory mediators were examined to investigate the mechanism by which CA inhibited LPS-induced inflammation. As presented in Fig. 4A and B, the levels of TLR4 and $N F-\kappa B$ mRNA and protein expressions in the LPS-treated group were significantly increased compared with the levels in the control group. However, the ability of LPS to promote the expression of TLR4 and NF- $\kappa \mathrm{B}$ mRNA, and protein was significantly inhibited by pretreatment with CA. Additionally, it was observed that the phosphorylation level of NF- $\kappa \mathrm{B}$ was significantly increased by LPS treatment and that this increase was inhibited by CA pretreatment in a dose-dependent manner (Fig. 4B). 
Subsequently, immunohistochemistry was used to examine the expression of TLR 4 and $\mathrm{NF}-\kappa \mathrm{B}$ in lung tissue. As presented in Fig. 4C, lung tissue from the control mice displayed very little positive staining for TLR4 and p65. After LPS treatment, the levels of TLR4 and p65 expression were significantly increased in the lung tissue epithelial cells. The LPS-induced increases in TLR4 and p65 were not significantly inhibited by pretreatment with $\mathrm{CA}$.

\section{Discussion}

Inflammation has been traditionally considered to be an important defense response induced by an infection or injury (27). However, inflammation can induce severe damage to various organs, including kidney (28), liver (29) and lungs (30). The use of drugs derived from plants, such as CA from Salvia officinalis, has been revealed to be beneficial in reversing inflammation-induced tissue injuries (17). In the present study, the effect of CA on LPS-induced inflammation and ALI was investigated in a mouse model of lung injury induced by LPS. This model is commonly used to determine the effectiveness of anti-inflammatory agents (31). The results of the present study demonstrated that CA significantly attenuated LPS-induced inflammation and injuries in lungs.

$\mathrm{CA}$ is a major bioactive component of rosemary extracts. The extensive use of rosemary extracts in medicines has verified the strong pharmaceutical activity of CA $(23,32,33)$. Recent studies identified the tissue-protective role of CA in several types of organ injury, including Parkinson's disease, colitis and acute myocardial injury $(22,32,33)$. The protective effect of CA is mainly ascribed to its anti-inflammatory and anti-oxidation properties (34). It has been revealed that CA treatment can inhibit the infiltration of inflammatory cells, including macrophages and neutrophils $(33,35)$. Consistent with that report, the decreased infiltration of inflammatory cells was observed in the present study and this was further supported by reduced levels of MPO activity, a neutrophil marker. The initiation and development of ARDS can be attributed to inflammatory cells, such as macrophages and neutrophils (36). In response to LPS exposure, alveolar macrophages become activated and subsequently induce neutrophil infiltration (36). Infiltrated inflammatory cells produce large quantities of reactive oxygen species (ROS) that exacerbate inflammatory responses and significantly contribute to ALI (37). CA exerts an antioxidant effect by scavenging ROS and promoting the generation of secondary anti-oxidants (34). Neutrophils participate in ALI largely due to their production of proinflammatory cytokines, including TNF- $\alpha$, that delay apoptosis (38). Neutrophils normally serve an important role in body defense; however, the long-term survival of neutrophils at inflammatory sites can aggravate tissue damage (39). During an inflammatory response, neutrophil clearance, achieved via efficient apoptosis, is the primary mechanism that limits lung injuries and promotes the dissipation of inflammation (40). A previous study has shown that delayed neutrophil apoptosis, prolonged neutrophil survival times and the continuous release of cytotoxic factors (including oxygen free radicals) by neutrophils into lung tissue result in lung injuries (41). The results of the present study demonstrated that injection of LPS caused significant lung injuries in rats and this significantly reduced the apoptosis of neutrophils in BALF, indicating that the delayed apoptosis of neutrophils contributed to lung injury in the rats and could potentially be alleviated by increasing the apoptosis rate of neutrophils. In addition to inhibiting inflammatory cell infiltration, the anti-inflammatory activity of CA may also be due to an increase in apoptosis in LPS-treated neutrophils, which may contribute to the CA protection of LPS-induced ALI.

The anti-inflammatory activity of CA can be attributed to its ability to inhibit the production of proinflammatory cytokines, which are increased during the early stages of ALI and are crucial for its occurrence and progression (42). A previous study demonstrated that CA inhibited the production of TNF- $\alpha$ in a macrophage cell line, RAW 264.7, stimulated with LPS (43). In addition, increasing evidence indicates that CA markedly inhibits the LPS-induced production of TNF-a, IL-1 $\beta$ and IL-6 in vitro $(43,44)$. In a previous study, CA was determined to inhibit the increased production of TNF- $\alpha$ and IL- 6 in rats with LPS-induced liver injuries and inhibit IL-1 $\beta$, IL-17A and IFN- $\gamma$ production in a rat model of colitis induced by dextran sulfate sodium (17). In the present study, it was determined that CA treatment significantly inhibited the LPS-induced increases of TNF-a, IL-6 and IL-1 $\beta$ levels. These cytokinesare secreted by the endothelium, epithelium and alveolar macrophages in response to an initial inflammatory insult (36). The current results indicated that $\mathrm{CA}$ inhibited the further exacerbation of endothelial and epithelial injuries induced by LPS, and thereby dampened the inflammatory process in ALI.

Several recent studies have demonstrated that the NF- $\mathrm{B}$ pathway serves a critical role in ALI (45-47). In the present study, after LPS treatment, NF- $\kappa \mathrm{B}$ is activated by TLR4, leading to increases in the production of TNF- $\alpha$, IL- $1 \beta$ and IL-6. These cytokines activate NF- $\mathrm{B}$ to initiate a cycle that broadens the original immune response (42). It has been revealed that $\mathrm{CA}$ inhibits $\mathrm{NF}-\kappa \mathrm{B}$ activation and the downstream signaling process, leading to reduced levels of TNF- $\alpha$, IL-1 $\beta$ and IL-6 (48). Furthermore, CA has also been demonstrated to block signaling pathways upstream of $\mathrm{NF}-\kappa \mathrm{B}$, including the Syk/Src, PI3K and Akt pathways (45). Consistent with a previous study (49), the results of the current study indicated that $\mathrm{NF}-\kappa \mathrm{B}$ activation was markedly increased in LPS-induced lung injury. Although upstream signaling was not investigated in the present study, the results indicated that Syk/Src pathway inhibition may be involved in mediating the protective effect of CA, as Syk has been demonstrated to be a direct enzymatic target of CA (45). The present study also revealed that TLR4 expression was significantly increased in LPS-injured lung tissue, which was significantly inhibited after treatment with $\mathrm{CA}$, indicating that the inflammatory response is limited by inhibiting the upstream targets of TLR4. Consistent with the current results, a previous study reported that CA exerted an inhibitory effect on the expression and phosphorylation of $\mathrm{NF}-\kappa \mathrm{B}$, as well as on the expression of TLR4 in various inflammatory disorders, including diabetes (50). Another study reported that $\mathrm{CA}$ induced cytotoxicity in breast cancer cells (51). In the current study, no toxic effects of CA were detected and no mortality occurred in all CA-treatment groups were equal. However, further studies are required to determine whether repeated long-term administration of CA produces toxic effects. 
In summary, the current results indicated that CA alleviated the injury of ALI by regulating immune responses and this regulation may result from the ability of CA to inhibit the expression of TLR4 and the phosphorylation of NF- $\kappa \mathrm{B}$. These results strongly indicated that $\mathrm{CA}$ may be a promising agent for the treatment of ARDS.

\section{Acknowledgements}

Not applicable.

\section{Funding}

The current study was supported by the Science and Technology Support Program of Suqian (grant no. S201628).

\section{Availability of data and materials}

The datasets used and/or analyzed during the current study are available from the corresponding author on reasonable request.

\section{Authors' contributions}

QL conceived and designed the present study. LL performed the experiments. HS and $\mathrm{KC}$ completed data analysis and interpretation. QL and $\mathrm{KC}$ drafted and revised the manuscript. All authors read and approved the final manuscript.

\section{Ethics approval and consent to participate}

All experimental protocols involving animals were approved by the Ethics Committee of the Zhongda Hospital Southeast University (Nanjing, China).

\section{Patient consent for publication}

Not applicable.

\section{Competing interests}

The authors declare that they have no competing interests.

\section{References}

1. Han S and Mallampalli RK: The acute respiratory distress syndrome: From mechanism to translation. J Immunol 194 855-860, 2015.

2. Gonzales JN, Lucas R and Verin AD: The acute respiratory distress syndrome: Mechanisms and perspective therapeutic approaches Austin J Vasc Med 2: pii: 1009, 2015

3. Koh Y: Update in acute respiratory distress syndrome. J Intensive Care 2: 2, 2014.

4. Geboers DG, de Beer FM, Tuip-de Boer AM, van der Poll T, Horn J, Cremer OL, Bonten MJ, Ong DS, Schultz MJ and Bos LD Plasma suPAR as a prognostic biological marker for ICU mortality in ARDS patients. Intensive Care Med 41: 1281-1290, 2015.

5. Peng J, Wei D, Fu Z, Li D, Tan Y, Xu T, Zhou J and Zhang T: Punicalagin ameliorates lipopolysaccharide-induced acute respiratory distress syndrome in mice. Inflammation 38: 493-499, 2015

6. Bdeir K, Higazi AA, Kulikovskaya I, Christofidou-Solomidou M, Vinogradov SA, Allen TC, Idell S, Linzmeier R, Ganz T and Cines DB: Neutrophil alpha-defensins cause lung injury by disrupting the capillary-epithelial barrier. Am J Respir Crit Care Med 181: 935-946, 2010.
7. Cribbs SK and Martin GS: Stem cells in sepsis and acute lung injury. Am J Med Sci 341: 325-332, 2011.

8. Zhou X, Dai Q and Huang X: Neutrophils in acute lung injury. Front Biosci (Landmark Ed) 17: 2278-2283, 2012.

9. Zhu T, Wang DX, Zhang W, Liao XQ, Guan X, Bo H, Sun JY, Huang NW, He J, Zhang YK, et al: Andrographolide protects against LPS-induced acute lung injury by inactivation of NF- $\kappa B$. PLoS One 8: e56407, 2013.

10. Kawai T and Akira S: The role of pattern-recognition receptors in innate immunity: Update on Toll-like receptors. Nat Immunol 11: 373-384, 2010.

11. Murray LA, Knight DA, McAlonan L, Argentieri R, Joshi A, Shaheen F, Cunningham M, Alexopolou L, Flavell RA, Sarisky RT and Hogaboam CM: Deleterious role of TLR3 during hyperoxia-induced acute lung injury. Am J Respir Crit Care Med 178: 1227-1237, 2008.

12. Barsness KA, Arcaroli J, Harken AH, Abraham E, Banerjee A, Reznikov L and McIntyre RC: Hemorrhage-induced acute lung injury is TLR-4 dependent. Am J Physiol Regul Integr Comp Physiol 287: R592-R599, 2004.

13. Jeyaseelan S, Chu HW, Young SK, Freeman MW and Worthen GS: Distinct roles of pattern recognition receptors CD14 and Toll-like receptor 4 in acute lung injury. Infect Immun 73: 1754-1763, 2005

14. Kazemi S, Shirzad H and Rafieian-Kopaei M: Recent findings in molecular basis of inflammation and anti-inflammatory plants. Curr Pharm Des 24: 1551-1562, 2018.

15. Nardi GM, Farias Januario AG, Freire CG, Megiolaro F, Schneider K, Perazzoli MR, Do Nascimento SR, Gon AC, Mariano LN, Wagner G, et al: Anti-inflammatory activity of berry fruits in mice model of inflammation is based on oxidative stress modulation. Pharmacognosy Res 8 (Suppl 1): S42-S49, 2016.

16. Xiang Q, Liu Z, Wang Y, Xiao H, Wu W, Xiao C and Liu X: Carnosic acid attenuates lipopolysaccharide-induced liver injury in rats via fortifying cellular antioxidant defense system. Food Chem Toxicol 53: 1-9, 2013.

17. Li H, Sun JJ, Chen GY, Wang WW, Xie ZT, Tang GF and Wei SD: Carnosic acid nanoparticles suppress liver ischemia/reperfusion injury by inhibition of ROS, Caspases and NF- $\kappa$ B signaling pathway in mice. Biomed Pharmacother 82: 237-246, 2016.

18. Wang T, Takikawa Y, Satoh T, Yoshioka Y, Kosaka K, Tatemichi Y and Suzuki K: Carnosic acid prevents obesity and hepatic steatosis in ob/ob mice. Hepatol Res 41: 87-92, 2011.

19. Peng CH, Su JD, Chyau CC, Sung TY, Ho SS, Peng CC and Peng RY: Supercritical fluid extracts of rosemary leaves exhibit potent anti-inflammation and anti-tumor effects. Biosci Biotechnol Biochem 71: 2223-2232, 2007.

20. Shin HB, Choi MS, Ryu B, Lee NR, Kim HI, Choi HE, Chang J, Lee KT, Jang DS and Inn KS: Antiviral activity of carnosic acid against respiratory syncytial virus. Virol J 10: 303, 2013.

21. de Oliveira MR: The dietary components carnosic acid and carnosol as neuroprotective agents: A mechanistic view. Mol Neurobiol 53: 6155-6168, 2016.

22. Kocak C, Kocak FE, Akcilar R, Isiklar OO, Kocak H, Bayat Z, Simsek H, Taser F and Altuntas I: Molecular and biochemical evidence on the protective effects of embelin and carnosic acid in isoproterenol-induced acute myocardial injury in rats. Life Sci 147: 15-23, 2016.

23. Ma HJ, Huang XL, Liu Y and Fan YM: Sulfur dioxide attenuates LPS-induced acute lung injury via enhancing polymorphonuclear neutrophil apoptosis. Acta Pharmacol Sin 33: 983-990, 2012.

24. Livak KJ and Schmittgen TD: Analysis of relative gene expression data using real-time quantitative PCR and the 2(-Delta Delta C(T)) method. Methods 25: 402-408, 2001.

25. Grommes J and Soehnlein O: Contribution of neutrophils to acute lung injury. Mol Med 17: 293-307, 2011.

26. Li YY, Zhang GY, He JP, Zhang DD, Kong XX, Yuan HM and Chen FL: Ufm1 inhibits LPS-induced endothelial cell inflammatory responses through the NF- $\kappa \mathrm{B}$ signaling pathway. Int J Mol Med 39: 1119-1126, 2017

27. Chovatiya R and Medzhitov R: Stress, inflammation, and defense of homeostasis. Mol Cell 54: 281-288, 2014.

28. Ueda $\mathrm{N}$ and Takasawa $\mathrm{K}$ : Impact of inflammation on ferritin, hepcidin and the management of iron deficiency anemia in chronic kidney disease. Nutrients 10: pii: E1173, 2018.

29. Li S, Hong M, Tan HY, Wang N and Feng Y: Insights into the role and interdependence of oxidative stress and inflammation in liver diseases. Oxid Med Cell Longev 2016: 4234061, 2016. 
30. Bhargava R, Janssen W, Altmann C, Andrés-Hernando A Okamura K, Vandivier RW, Ahuja N and Faubel S: Intratracheal IL-6 protects against lung inflammation in direct, but not indirect, causes of acute lung injury in mice. PLoS One 8: e61405, 2013.

31. Chen $\mathbf{H}$, Bai $\mathbf{C}$ and Wang $\mathbf{X}$ : The value of the lipopolysaccharide-induced acute lung injury model in respiratory medicine. Expert Rev Respir Med 4: 773-783, 2010.

32. Lin CY, Chen JH, Fu RH and Tsai CW: Induction of Pi form of glutathione $\mathrm{S}$-transferase by carnosic acid is mediated through $\mathrm{PI} 3 \mathrm{~K} / \mathrm{Akt} / \mathrm{NF}-\kappa \mathrm{B}$ pathway and protects against neurotoxicity. Chem Res Toxicol 27: 1958-1966, 2014.

33. Yang N, Xia Z, Shao N, Li B, Xue L, Peng Y, Zhi F and Yang Y: Carnosic acid prevents dextran sulfate sodium-induced acute colitis associated with the regulation of the Keap1/Nrf2 pathway. Sci Rep 7: 11036, 2017.

34. Xia G, Wang X, Sun H, Qin Y and Fu M: Carnosic acid (CA) attenuates collagen-induced arthritis in $\mathrm{db} / \mathrm{db}$ mice via inflammation suppression by regulating ROS-dependent p38 pathway. Free Radic Biol Med 108: 418-432, 2017.

35. Osakabe N, Yasuda A, Natsume $M$ and Yoshikawa $T$ : Rosmarinic acid inhibits epidermal inflammatory responses: Anticarcinogenic effect of Perilla frutescens extract in the murine two-stage skin model. Carcinogenesis 25: 549-557, 2004.

36. Williams AE and Chambers RC: The mercurial nature of neutrophils: Still an enigma in ARDS? Am J Physiol Lung Cell Mol Physiol 306: L217-L230, 2014.

37. Dong Z and Yuan Y: Accelerated inflammation and oxidative stress induced by LPS in acute lung injury: Inhibition by ST1926. Int J Mol Med 41: 3405-3421, 2018.

38. Lin WC, Lin CF, Chen CL, Chen CW and Lin YS: Inhibition of neutrophil apoptosis via sphingolipid signaling in acute lung injury. J Pharmacol Exp Ther 339: 45-53, 2011.

39. Selders GS, Fetz AE, Radic MZ and Bowlin GL: An overview of the role of neutrophils in innate immunity, inflammation and host-biomaterial integration. Regen Biomater 4: 55-68, 2017.

40. Lee WL and Downey GP: Neutrophil activation and acute lung injury. Curr Opin Crit Care 7: 1-7, 2001.

41. Zhenglong Y, Qiaolian X and Haibo Q: Role of neutrophil apoptosis in the lung of rats with acute lung injury. Chin J Crit Care Med 25: 503-505, 2005

42. Zhang X, Shang F, Hui L, Zang K and Sun G: The alleviative effects of metformin for lipopolysaccharide-induced acute lung injury rat model and its underlying mechanism. Saudi Pharm J 25: 666-670, 2017.
43. Kuo CF, Su JD, Chiu CH, Peng CC, Chang CH, Sung TY, Huang SH, Lee WC and Chyau CC: Anti-inflammatory effects of supercritical carbon dioxide extract and its isolated carnosic acid from Rosmarinus officinalis leaves. J Agric Food Chem 59: 3674-3685, 2011

44. Yang T, Zhang J, Sun L, Zhu X, Li J, Wang J, Chen H, Bao R, Deng X, Hou J and Liu Y: Combined effects of a neutrophil elastase inhibitor (sivelestat sodium) and a free radical scavenger (edaravone) on lipopolysaccharide-induced acute lung injury in rats. Inflamm Res 61: 563-569, 2012.

45. Liu SF and Malik AB: NF-kappa B activation as a pathological mechanism of septic shock and inflammation. Am J Physiol Lung Cell Mol Physiol 290: L622-L645, 2006.

46. Everhart MB, Han W, Sherrill TP, Arutiunov M, Polosukhin VV, Burke JR, Sadikot RT, Christman JW, Yull FE and Blackwell TS: Duration and intensity of NF-kappaB activity determine the severity of endotoxin-induced acute lung injury. J Immunol 176: 4995-5005, 2006.

47. Liu S, Feng G, Wang GL and Liu GJ: p38MAPK inhibition attenuates LPS-induced acute lung injury involvement of NF-kappaB pathway. Eur J Pharmacol 584: 159-165, 2008.

48. Tang B, Tang F, Wang Z, Qi G, Liang X, Li B, Yuan S, Liu J, $\mathrm{Yu}$ S and $\mathrm{He} \mathrm{S}$ : Upregulation of Akt/NF- $\mathrm{BB}$-regulated inflammation and Akt/Bad-related apoptosis signaling pathway involved in hepatic carcinoma process: Suppression by carnosic acid nanoparticle. Int J Nanomedicine 11: 6401-6420, 2016.

49. Oh J, Yu T, Choi SJ, Yang Y, Baek HS, An SA, Kwon LK, Kim J, Rho HS, Shin SS, et al: Syk/Src pathway-targeted inhibition of skin inflammatory responses by carnosic acid. Mediators Inflamm 2012: 781375, 2012

50. Park MY and Mun ST: Carnosic acid inhibits TLR4-MyD88 signaling pathway in LPS-stimulated 3T3-L1 adipocytes. Nutr Res Pract 8: 516-520, 2014

51. Yildiz-Ozturk E, Gulce-Iz S, Anil M and Yesil-Celiktas O: Cytotoxic responses of carnosic acid and doxorubicin on breast cancer cells in butterfly-shaped microchips in comparison to 2Dand 3D culture. Cytotechnology 69: 337-347, 2017.

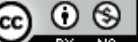

This work is licensed under a Creative Commons Attribution 4.0 International (CC BY-NC 4.0) License 